\title{
Human immune recognition-based multicomponent subunit vaccines against
} tuberculosis

\author{
S.B. Sable*, I. Verma*, D. Behera ${ }^{\#}$ and G.K. Khuller*
}

ABSTRACT: The cell-mediated immune response, with its shift in favour of type-1 over type-2 Thelper cell immune response, is generally regarded as essential to protection against mycobacterial infections. The aim of this study was to evaluate the protective potential of two multicomponent subunit vaccines (MSV-1 and MSV-2) against tuberculosis (TB) based on human immune recognition.

MSV-1 consisted of five immunodominant antigens (TB10.4, early secretory antigenic target (ESAT)-6, culture filtrate protein (CFP)-8, CFP-10 and CFP-15) selected from a group of polypeptides, which induced a predominant T-cell response in immune human subjects, whereas MSV-2 consisted of antigens (CFP-11, CFP-21, CFP-22.5, Mycobacterium tuberculosis protein (MPT)-64 and CFP-31) selected from a group of polypeptides which induced a subdominant T-cell response along with the antibody response.

Both of these sets of polypeptides were extensively recognised in healthy individuals with significant interferon gamma release compared to the diseased population. In C57BL/6J mice, at the level of the lungs, the order of protective efficacy for the test vaccines was: bacille CalmetteGuérin (BCG) $>$ MSV-2 $>$ MSV-1. The protective efficacy of MSV-1 was found to be significantly less than that of MSV-2 and BCG at the level of spleen, whereas that of MSV-2 was comparable to that of BCG.

The results of this study indicate that high T-helper cell type 1 response-inducing polypeptides selected on the basis of human immune recognition do not necessarily impart protection during vaccination experiments.

KEYWORDS: Human immunity, multicomponent subunit vaccine, tuberculosis

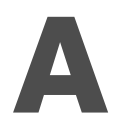
subunit vaccine consisting of key protective antigens of Mycobacterium tuberculosis could have advantages over the existing bacille Calmette-Guérin (BCG) vaccine. Since the early 1990s, efforts to develop a subunit vaccine against tuberculosis (TB) have focused on proteins released from the growing mycobacteria into the extracellular medium [1-3]. Many are unique to M. tuberculosis, and, to date, only a few have been evaluated for their immunological properties and protective potential in various animal models as subunit vaccine candidates [38]. Furthermore, the majority of the antigens used to date for vaccine purposes have been selected on the basis of immunoreactivity in animal models $[3,6,7]$. However, ideal vaccine antigens need to be selected on the basis of immune recognition by a large percentage of different ethnic human populations [9-11]. Moreover, the immunological parameters required for protective efficacy of an antituberculous vaccine are still not clearly defined. Further, as a vaccine based on a single antigen cannot be consistently protective in a genetically diverse population, experimental vaccines based on the combination of protective antigens also need to be evaluated [8, 12].

The low molecular mass protein fractions isolated from the secretory proteome of $<40 \mathrm{kDa}$ of M. tuberculosis are known to be predominantly recognised by the peripheral blood mononuclear cells (PBMCs) of healthy TB contacts, a human model of protective immunity to TB $[9,11]$. Therefore, in the present study, a group of immunodominant low molecular mass purified polypeptides predominantly recognised by $\mathrm{T}$ lymphocytes and another group of immunodominant antigens recognised by both the T- and B-lymphocytes of immune subjects (TB contacts / memory immune) were evaluated for their recognition by different donor categories of

\section{AFFILIATIONS}

Depts of *Biochemistry and \#Pulmonary Medicine, Postgraduate Institute of Medical Education \& Research, Chandigarh, India.

\section{CORRESPONDENCE}

G.K. Khuller

Dept of Biochemistry

Postgraduate Institute of Medical

Education \& Research

Chandigarh

160012

India

Fax: 911722744401

E-mail: gkkhuller@yahoo.co.in

Received:

September 092004

Accepted after revision:

December 092004

SUPPORT STATEMENT

This study was funded by a grant from the Dept of Biotechnology and Indian Council of Medical Research of the Government of India (New Delhi, India). S.B. Sable is the recipient of a Senior Research Fellowship of the Council of Scientific and Industrial Research (New Delhi, India). 
human TB and healthy subjects. Two experimental multicomponent subunit vaccines (MSVs) constituted using highly immunodominant polypeptides from both groups were evaluated for their protective efficacy against experimental TB in C57BL/6J mice using dimethyldioctadecylammonium bromide (DDA)-monophosphoryl lipid A (MPL) adjuvants.

\section{MATERIALS AND METHODS Bacterial culture}

M. tuberculosis strain $\mathrm{H} 37 \mathrm{Rv}$, originally obtained from the National Collection of Type Cultures (London, UK) and maintained on Löwenstein-Jensen medium, was used in the present study.

\section{Animals}

Female 4-5-week-old C57BL/6J (H-2 $\left.{ }^{\mathrm{b}}\right)$ mice weighing 15-20 g (National Centre for Laboratory Animal Sciences, Hyderabad, India) were used in the present study. Mice were housed in cages contained within a negative pressure-regulated animal isolator and were fed on a standard pellet diet and water ad libitum.

\section{Study population}

Newly diagnosed moderately advanced TB patients (13 males (mean $\pm S D$ aged $45 \pm 10 \mathrm{yrs}$ ); five females (aged $14 \pm 8 \mathrm{yrs}$ )) who were admitted to the Nehru Hospital (Postgraduate Institute of Medical Education \& Research, Chandigarh, India) and the Tuberculosis and Chest Diseases Hospital (Patiala, India) formed part of the study population. The diagnosis was based on history and chest radiography, and the extent of the disease was graded by chest radiography according to the criteria of the National Tuberculosis and Respiratory Disease Association (New York, NY, USA) [13]. All of the patients had a history of cough, fever and cachexia lasting $>6$ months and their chest radiographs were suggestive of $\mathrm{TB}$. The active disease was further confirmed by sputum smear positivity for acid-fast bacilli using Ziehl-Neelsen staining. All patients demonstrated more than two bacteria in 10 fields in repeated smear tests. All of the patients were later found to respond to antituberculous therapy. Healthy medical and paramedical subjects (12 males (aged $34 \pm 6$ yrs); 11 females ( $29 \pm 5$ yrs)), who were in direct contact with TB patients or in contact with biological samples or cultures from these patients for $>6$ months, were considered to be hospital contacts and were drawn from the above-mentioned hospitals. All TB contacts and endemic controls had no previous history of TB. Active pulmonary TB was excluded from TB contacts and endemic controls by chest radiography and sputum smears for acid-fast bacilli. Moreover, all contacts and endemic controls remained healthy over a period of 3 yrs. The subclinical infection of TB contacts was confirmed by early secretory antigenic target (ESAT)-6-specific in vitro lymphoproliferation and tuberculin skin test positivity. Eighty-two per cent of TB contacts showed ESAT-6-specific lymphoproliferation (stimulation index (SI) $>3$ ) and all TB contacts were positive for Siebert purified protein derivative of tuberculin (PPD) with a median induration response of $20 \mathrm{~mm}$ and minimum induration readings of $15 \mathrm{~mm}$ in the Mantoux test. Recently admitted graduate students from different socio-economic strata (nine males (aged $25 \pm 2$ yrs); eight females (aged $24.5 \pm 2$ yrs)) served as endemic controls and were all healthy with no known contact with TB patients. The median induration response of endemic controls was $13 \mathrm{~mm}$, with $29 \%$ showing ESAT-6-specific in vitro lymphoproliferation and $24 \%$ exhibiting induration readings of $>15 \mathrm{~mm}$ in the Mantoux test. All diseased and healthy subjects used in the present study were adults who had received childhood $M$. bovis BCG vaccination, but without any booster dose, and were also HIV-negative. The Institutional Ethics Committee of the Postgraduate Institute of Medical Education \& Research approved the study, and blood samples were drawn with the prior consent of the study subjects.

\section{Antigens}

Total culture filtrate proteins (CFPs) of M. tuberculosis $\mathrm{H} 37 \mathrm{Rv}$ (RvCFP) were prepared by growing $M$. tuberculosis in stationary pellicle culture in modified liquid Youman's medium for 4 weeks [14]. A total of 26 low molecular mass polypeptides identified as immunodominant antigenic targets from $M$. tuberculosis H37Rv culture filtrate and categorised into different groups on the basis of type of immune response induced in humans models of immunity to TB were used as test antigens. The polypeptides were purified using a strategy based on separation of complex mixtures of secretory proteins on the basis of charge using diethylaminoethyl-Sepharose CL$6 \mathrm{~B}$ anion exchange column chromatography as a first step [15], followed by separation according to size via high-resolution preparative sodium dodecylsulphate-polyacrylamide gel electrophoresis and subsequent electroelution [10].

\section{Isolation of PBMCs and lymphocyte proliferation assay}

PBMCs were isolated from heparinised venous blood samples by density-gradient centrifugation using Ficoll-Hypaque. The cells were cultured at $1 \times 10^{5}$ cells well $^{-1}$ in complete RPMI 1640 medium (Sigma, St Louis, MO, USA) [11]. Purified low molecular mass polypeptides $\left(2 \mu \mathrm{g} \cdot \mathrm{mL}^{-1}\right)$ were used for in vitro stimulation. RvCFP and PPD $\left(2 \mu \mathrm{g} \cdot \mathrm{mL}^{-1}\right)$ were used for comparison, whereas phytohaemagglutinin $\left(1 \mu \mathrm{g} \cdot \mathrm{mL}^{-1}\right)$ was used as mitogen to check cell reactivity and viability.

\section{Interferon- $\gamma$ ELISA}

Interferon (IFN)- $\gamma$ ELISA was carried out to estimate the IFN- $\gamma$ levels released in lymphocyte culture supernatants on day 5 in response to in vitro stimulation with antigens using a commercially available anti-human IFN- $\gamma$ reagent set (OPT EIA $^{\mathrm{TM}}$; Becton Dickinson and Company, Biosciences, Pharmingen, San Diego, CA, USA). The assay was performed according to the manufacturer's instructions. The detection limit of the assay was $2.35 \mathrm{pg} \cdot \mathrm{mL}^{-1}$.

\section{Cytotoxicity assay}

The in vitro cytotoxic T-lymphocyte (CTL) response induced by mycobacterial antigens was measured by means of neutral red assay as described by PARISH and MULLBACHER [16] with some modifications. Monocytes obtained as adherent populations were cultured in 96-well flat-bottomed tissue culture plates in

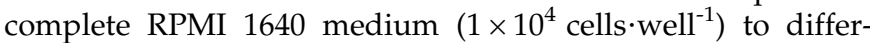
entiate into macrophages (target cells), whereas total PBMCs $\left(1 \times 10^{6}\right.$ cells $\left.\cdot \mathrm{mL}^{-1}\right)$ were cultured with $20 \mu \mathrm{g} \cdot \mathrm{mL}^{-1} \mathrm{RvCFP}$ in 6well plates in a humidified atmosphere of $5 \%$ carbon dioxide at $37^{\circ} \mathrm{C}$ for 7 days to obtain effector cells. On day 7, differentiated macrophages were gently washed in RPMI 1640 medium and cultured overnight with the optimal concentration of purified 
mycobacterial antigens ( $2 \mu \mathrm{g}$ purified polypeptides). On day 8 , nonadherant effector cells from antigen-stimulated PBMC cultures were harvested and counted. Effector cells were dispensed in a 96-well plate containing macrophages pulsed with antigen at the optimal effector:target cell ratio of 20:1. Effector and target cells were co-cultured in RPMI 1640 medium containing $10 \%$ autologous serum for $16 \mathrm{~h}$. At the end of the incubation period, the wells were washed and the remaining adherent cells incubated with $0.04 \%$ neutral red in PBS (pH 7.4) for $1 \mathrm{~h}$, and finally washed in RPMI 1640/PBS. A solution of $0.1 \mathrm{M}$ acetic acid in $50 \%$ ethanol was added to each well to release neutral red. Absorbance was read at $550 \mathrm{~nm}$, and results were expressed as percentage lysis of target cells at the effector:target cell ratio used.

\section{Immunisation and challenge of mice}

Mice were immunised with two sets of cocktail preparations, each containing five immunodominant antigens selected on the basis of human immune recognition studies. Cocktail preparation $(0.2 \mathrm{~mL})$ was injected subcutaneously on the back, in three equal doses of $50 \mu \mathrm{g}$ (containing $10 \mu \mathrm{g}$ of each antigen) at biweekly intervals, using MPL and DDA (both Sigma) as adjuvants. Antigens were emulsified in DDA $\left(250 \mu \mathrm{g} \cdot \mathrm{dose}^{-1}\right)$, and MPL was used as coadjuvant $\left(25 \mu \mathrm{g} \cdot \mathrm{dose}^{-1}\right)$ as described previously [4]. At the time of the last dose of experimental subunit vaccination, a group of mice received a single dose of BCG (BCG Vaccine Laboratory, Guindy, Chennai, India; $1 \times 10^{5}$ colony-forming units (cfu)) injected subcutaneously at the base of the tail. The mice were challenged 8 weeks after the first dose of experimental MSV preparation via the lateral tail vein with $1 \times 10^{5} \mathrm{cfu} M$. tuberculosis $\mathrm{H} 37 \mathrm{Rv}$ suspended in
$0.1 \mathrm{~mL}$ PBS. Four weeks after the challenge, the animals were sacrificed and M. tuberculosis cfu enumerated in target organs [4].

\section{Statistical methods}

In the case of human recognition experiments, comparison between different groups of individuals was performed using the Mann-Whitney two-tailed test. For protection experiments, two-way comparison between the test and control group was performed using an unpaired t-test. Multiple comparisons between different groups were performed by means of ANOVA. The statistical analyses were considered significant at the level of $\mathrm{p}<0.05$.

\section{RESULTS}

Immune responses of healthy TB contacts and TB patients to mycobacterial antigens

Polypeptides (104 electrophoretic bands) were purified from the low-molecular-mass region $(<40 \mathrm{kDa})$ of the secretory proteome of $M$. tuberculosis. The purified polypeptides were subjected to recognition testing by PBMCs from healthy TB contacts and healed TB patients (data not shown), a population considered as a model of protective immunity against TB [911]. The 28 polypeptides selected on the basis of initial screening in the immune population were used to constitute groups I (table 1) and II (table 2). All group-I and group-II antigens were initially subjected to reactivity testing with a panel of known monoclonal antibodies (mAbs) or polyclonal antibodies in order to identify previously defined CFPs of low molecular mass by ELISA. The antibodies CS-18 (directed against superoxide dismutase), $\alpha$-MPT-53 (M. tuberculosis

\section{TABLE 1 Characterisation of group-I purified polypeptides}

\begin{tabular}{|c|c|c|c|c|c|c|c|}
\hline Serial No. & Protein No. & mAb reactivity & H37Rv annotation & App $M_{r}$ kDa & Theor $\mathbf{M}_{\mathbf{r}}^{\#} \mathbf{k D a}$ & pl & Identity \\
\hline 1 & 6 & Nil & Unknown & 4.5 & Unknown & Unknown & Unknown \\
\hline 2 & 35 & PV-2++ & Unknown & 4.0 & Unknown & Unknown & ESAT- 6 family member? ${ }^{+}$ \\
\hline 3 & 36 & $P V-2+++++$ & Rv0288 & 4.5 & 10.391 & 4.50 & TB10.4 $4^{+}$ \\
\hline 4 & 38 & HYB 76-8+++++ & Rv3875 & 6.0 & 11.75 & 3.52 & ESAT- $6^{+, \S}$ \\
\hline 5 & 39 & HYB 76-8++ & Unknown & 7.0 & Unknown & Unknown & ESAT-6 family member? ${ }^{+}$ \\
\hline 6 & 40 & Nil & Rv0496 & 8.0 & 34.80 & 6.32 & CFP-8 (conserved hypothetical protein) $)^{\uparrow, \S}$ \\
\hline 7 & 41 & K8493++ & Unknown & 9.0 & Unknown & Unknown & ESAT-6 family member?+ \\
\hline 8 & 42 & K8493+++ & Unknown & 9.5 & Unknown & Unknown & ESAT-6 family member? ${ }^{+}$ \\
\hline 9 & 43 & K8493+++++ & Rv3874 & 10.0 & 10.79 & 4.31 & CFP $-10^{+}$ \\
\hline 10 & 44 & SA-12/IT-3++ & Unknown & 11.0 & Unknown & Unknown & Unknown \\
\hline 11 & 47 & $\alpha$-MPT-53++ & Rv0164 & 15.0 & 20.18 & 5.90 & CFP $-15^{\uparrow, \S}$ \\
\hline 12 & 65 & Nil & Unknown & 5.5 & Unknown & Unknown & Unknown \\
\hline 13 & 66 & Nil & Unknown & 6.0 & Unknown & Unknown & Unknown \\
\hline 14 & 69 & SA-12/IT-3+++ & Unknown & 9.0 & Unknown & Unknown & Unknown \\
\hline 15 & 70 & SA-12/IT3+++++ & Rv3418C & 10.0 & 14.45 & 4.93 & GroES (hsp10/MPT-57) ${ }^{+, \S}$ \\
\hline 16 & 76 & $\alpha-\mathrm{MPT}-53++$ & Rv2445C & 14.5 & 14.47 & 5.30 & CFP-14.5 (ndkA) ${ }^{\varphi, \S}$ \\
\hline 17 & 77 & Nil & Rv1827 & 16.0 & 17.21 & 4.30 & CFP $-16^{\bullet, \S}$ \\
\hline 18 & 79 & Nil & Rv2185C; Rv1827 & 18.0 & $16.292 ; 17.21$ & $4.80 ; 4.30$ & CFP-18 (mixed sample) ${ }^{\S}$ \\
\hline
\end{tabular}

These polypeptides induced a predominant T-cell response in human subjects with protective immunity to tuberculosis (TB). mAb: monoclonal antibody; App: apparent; $\mathrm{M}_{\mathrm{r}}$ : relative molecular mass; Theor: theoretical; pl: isoelectric point; Nil: no reactivity; ESAT: early secretory antigenic target; CFP: culture filtrate protein; GroES: 10-kDa heat shock protein (hsp10); MPT: Mycobacterium tuberculosis protein; ndk: nucleoside diphosphate kinase. ++: optical density (OD) 0.20-0.30; +++: OD 0.30-0.40; +++++: OD>0.50. \#: from [17]; ${ }^{\bullet}$ : novel T-cell antigen; ${ }^{+}$: by mAb/polyclonal antibody reactivity; ${ }^{\S}$ : by liquid chromatography-tandem mass spectrometry. 


\section{TABLE 2 Characterisation of group-II purified polypeptides}

\begin{tabular}{|c|c|c|c|c|c|c|c|}
\hline Serial No. & Protein No. & mAb reactivity & H37Rv annotation & App $M_{r} k D a$ & Theor $\mathbf{M}_{\mathbf{r}}^{\#} \mathbf{k D a}$ & pl & Identity \\
\hline 1 & 1 & K8483++ & Rv0632C; Rv1626 & 22.5 & $24.35 ; 22.66$ & $5.50 ; 5.00$ & $\begin{array}{l}\text { CFP-22.5 (mixed sample: enoyl-CoA- } \\
\text { hydratase; component response regulator) }\end{array}$ \\
\hline 2 & 2 & K8483+++++ & Rv1984C & $21.0-22.0$ & 18.67 & 4.60 & CFP-21 (cutinase precursor) ${ }^{\S}$ \\
\hline 3 & 25 & Nil & Unknown & 19.0 & Unknown & Unknown & Unknown \\
\hline 4 & 28 & L24b4+++++ & Rv1980C & 24.0 & 26.15 & 4.83 & MPT- $64^{+, \S, f}$ \\
\hline 7 & 33 & Nil & Rv0831C & 31.0 & 30.18 & 5.10 & CFP-31 (hypothetical protein) ${ }^{\text {f,+ }}$ \\
\hline 8 & 37 & PV-2; HYB 76-8++ & Unknown & 5.5 & Unknown & Unknown & ESAT-6 family member? ${ }^{\S}$ \\
\hline 9 & 45 & IT-3/SA-12++ & Rv3874; Rv3592; Rv3914 & 12.5 & $10.00 ; 11.00 ; 12.50$ & $5.11(\operatorname{Rv} 3914)$ & $\begin{array}{l}\text { CFP-12.5" (mixed sample: } 10 \text { and } 11 \mathrm{kDa} \\
\text { conserved hypothetical protein; } 12.5 \mathrm{kDa} \\
\text { thioredoxin/MPT-46) }{ }^{+}\end{array}$ \\
\hline 10 & 46 & $\mathrm{Nil}$ & Rv2433C & 13.5 & 11.00 & 10.93 & CFP-11 (conserved hypothetical protein) ${ }^{\bullet, f}$ \\
\hline
\end{tabular}

These polypeptides induced both $\mathrm{T}$ - and D-cell responses in human subjects with protective immunity to tuberculosis. mAb: monoclonal antibody; App: apparent; $\mathrm{M}_{\mathrm{r}}$ relative molecular mass; Theor: theoretical; pl: isoelectric point; Nil: no reactivity; CFP: culture filtrate protein; CoA: coenzyme A; MPT: Mycobacterium tuberculosis protein; Ag: antigen; ESAT: early secretory antigenic target. ++: optical density (OD) 0.20-0.30; ++1++: OD>0.50. \#: from [17]; ": novel T-cell antigen; ${ }^{+}:$by liquid chromatography-tandem mass spectrometry; ${ }^{\text {s. }}$ by $\mathrm{mAb} /$ polyclonal antibody reactivity; ${ }^{f}$ : by $\mathrm{N}$-terminal sequencing.

protein (MPT) 53), IT-3 (10-kDa heat shock protein (hsp 10)), IT-4 (16-kDa $\alpha$-crystallin), IT-20 (14-kDa $\alpha$-crystallin), IT-10 (20.5-kDa uncharacterised protein), IT-12 (19-kDa lipoprotein), IT-23 (phosphate transport subunit S), IT-44 (CFP-32), IT-49 (antigen (Ag) 85 complex), IT-52 (MPT-51), IT-59 (33-kDa uncharacterised protein), IT-69 (CFP-20), mc9246 (28-kDa uncharacterised protein), PV-2 (TB10.4), HYB 76-8 (ESAT-6), L24b4 (MPT-64), K8483 (CFP-21) and K8493 (CFP-10) were used as probes. Subsequently, selected immunodominant antigens were further characterised by either N-terminal sequencing or liquid chromatography-tandem mass spectrometry (tables 1 and 2). These immunodominant polypeptides were subjected to recognition testing by peripheral blood lymphocytes of various donor categories in the present study. Among all the donors, healthy contacts demonstrated high proliferative and IFN- $\gamma$ responses to the mycobacterial antigens tested, whereas TB patients gave exceedingly low responses. Considering an SI of 3.0 as the positive cut-off value for determining percentage recognition, all of the polypeptides of the two groups (I and II) were found to be predominantly recognised by PBMCs from healthy TB contacts (fig. $1 \mathrm{a}$ and $\mathrm{b}$ ). In group I, the maximum lymphocyte proliferation was observed in response to polypeptide 42 (median SI 16.87 (interquartile range (IQR) 5.46-25.19); 78.26\% recognition), whereas, in group II, the maximum lymphocyte proliferation was observed in response to polypeptide 45 (median SI 10.04 (IQR 4.26-14.77); 78.26\% recognition). The maximum IFN- $\gamma$ response was induced by polypeptide 43 (median [IFN- $\gamma$ ] $178.0 \mathrm{pg} \cdot \mathrm{mL}^{-1}$ (IQR 44.00-295.00 $\left.\mathrm{pg} \cdot \mathrm{mL}^{-1}\right)$ ) in group I, whereas, in group II, polypeptide 2 (median [IFN- $\gamma$ ] $170.0 \mathrm{pg} \cdot \mathrm{mL}^{-1}$ (IQR 39.70-298.00 $\left.\mathrm{pg} \cdot \mathrm{mL}^{-1}\right)$ ) was found to induce high IFN- $\gamma$ levels (fig. $1 \mathrm{c}$ and $\mathrm{d}$ ).

When the PBMC responses of moderately advanced TB patients (fig. 2) were compared to those of healthy TB contacts (fig. 1), suppressed responses towards mycobacterial antigens were observed. Analysis of lymphocyte proliferative responses obtained with group-I and group-II polypeptides indicated that only polypeptide Nos. 39 and 41 (median SI >3) were recognised by PBMCs of TB patients (fig. $2 a$ and b). The lymphocyte proliferation response, as well as IFN- $\gamma$ release, was maximum for polypeptide 39 (median SI 3.92 (IQR 2.18$5.54) ; 55.55 \%$ recognition; median IFN- $\gamma \quad 64.50 \mathrm{pg} \cdot \mathrm{mL}^{-1}$ (IQR $\left.18.25-153.75 \mathrm{pg} \cdot \mathrm{mL}^{-1}\right)$ ). The lymphocyte proliferative responses of all group-I polypeptides except for polypeptide 6 were significantly high in healthy TB contacts compared to moderately advanced TB patients. Conversely, except for the Ag85A and B complex, all group-II polypeptides were found to induce significantly pronounced lymphocyte proliferative responses in healthy TB contacts (fig. 1a and b; fig. $2 \mathrm{a}$ and b). The IFN- $\gamma$ responses of all group-I polypeptides except for polypeptides $6,42,66$ and 70 were significantly high in healthy TB contacts, whereas all of the group-II polypeptides except for polypeptide 25 demonstrated significantly high IFN- $\gamma$ responses in healthy TB contacts compared to moderately advanced TB patients (fig. 1c and d; fig. 2c and d).

According to prevailing concepts of TB vaccination, polypeptides predominantly recognised by T-lymphocytes of healthy TB contacts, but not by TB patients are implicated in protective immunity [9-11]. When group-I polypeptides were analysed for inclusion in an experimental MSV, polypeptides 35, 36, 38, $39,40,41,42,43,47$ and 65 were identified as the top 10 polypeptides predominantly recognised by T-lymphocytes of healthy TB contacts (when arbitrary cut-offs of median SI of $>5.0$ and median IFN- $\gamma$ level of $>50.0 \mathrm{pg} \cdot \mathrm{mL}^{-1}$ were employed). Polypeptide 42 induced marked lymphocyte proliferation and IFN- $\gamma$ induction in healthy TB contacts. However, it was also found to be recognised in TB patients, and was considered unsuitable for inclusion in experimental vaccine preparation. Polypeptides 35, 39, 41 and 42 crossreacted with $\mathrm{mAbs} /$ polyclonal antibodies used to identify 
a)

80
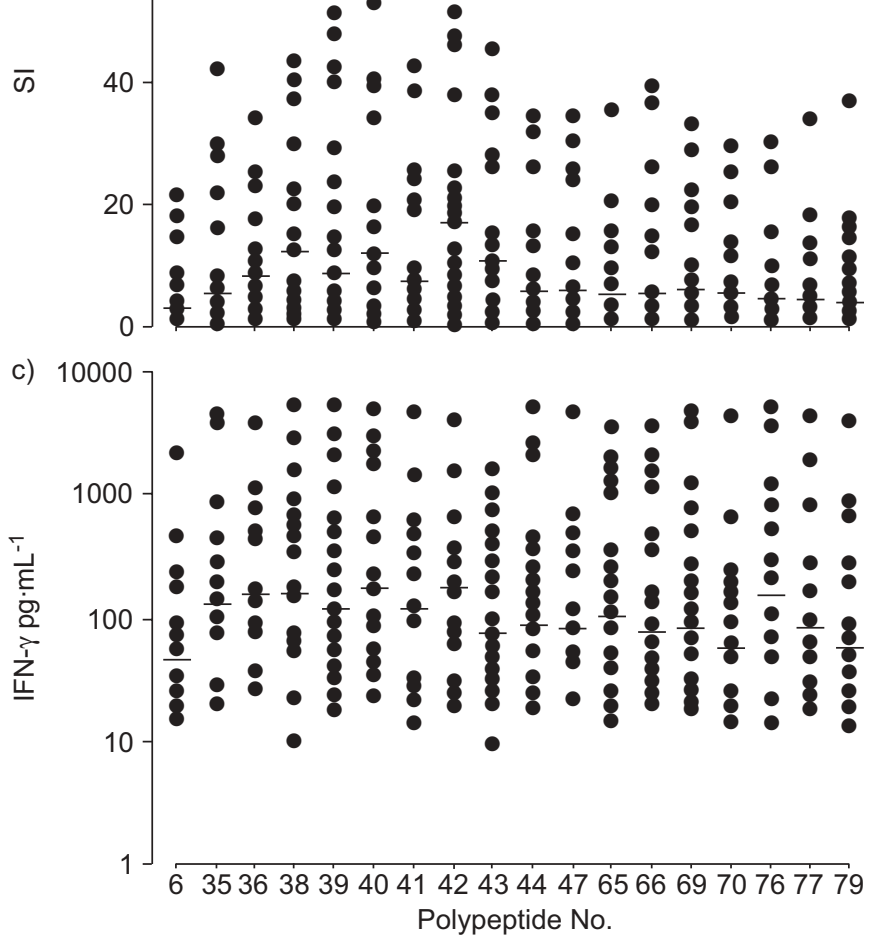

b)

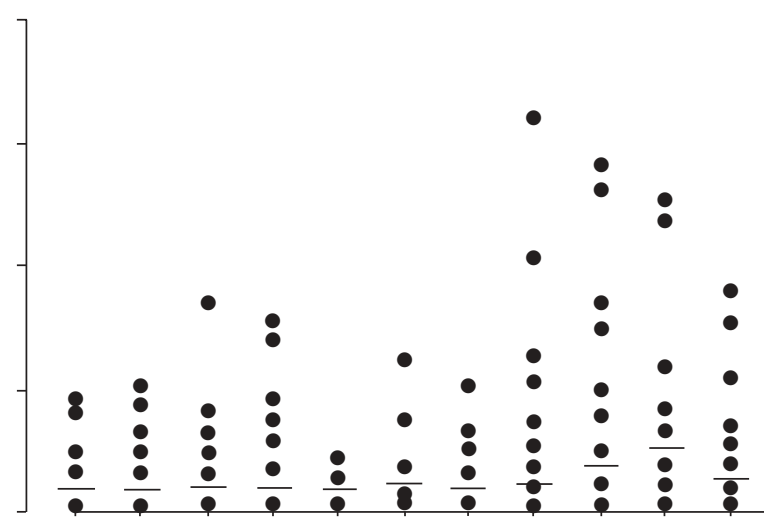

d)

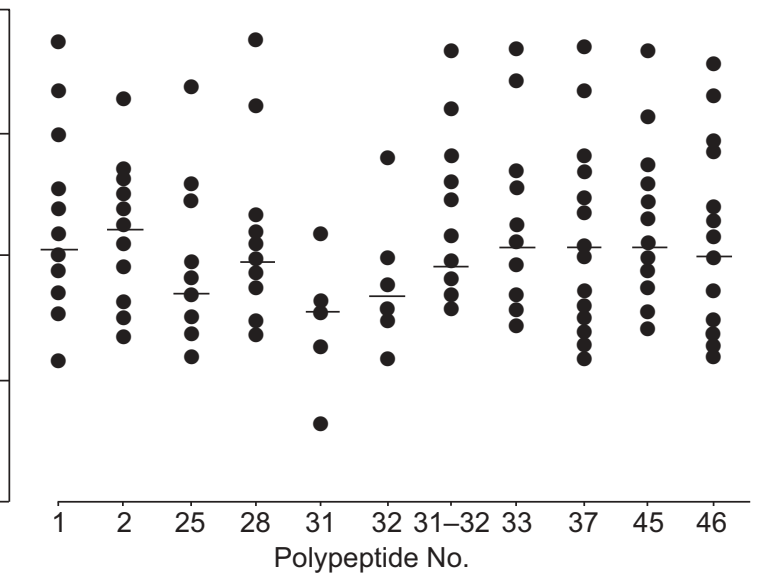

FIGURE 1. a, b) Lymphocyte proliferative and c, d) interferon (IFN)- $\gamma$ responses of healthy tuberculosis contacts ( $n=23$ ) after in vitro stimulation with: a, c) group-l, and b, d) group-II low molecular mass purified polypeptides. The median induration response in tuberculin skin tests carried out in the study subjects was $20 \mathrm{~mm}$. Horizontal bars represent medians and each symbol represents one individual. The median counts per minute of cultures without antigen was 873 . The median stimulation index (SI) in response to phytohaemagglutinin (PHA), total culture filtrate proteins of Mycobacterium tuberculosis H37Rv (RvCFP) and Siebert purified protein derivative of tuberculin (PPD) was 24.91 (interquartile range (IQR) 13.35-50.49), 8.56 (IQR 4.23-16.21) and 9.49 (IQR 5.49-15.66), respectively. The median IFN- $\gamma$ response of cultures without antigen was $12.0 \mathrm{pg} \cdot \mathrm{mL}^{-1}$. The IFN- $\gamma$ levels released into lymphocyte culture supernatants in response to in vitro stimulation with PHA, RvCFP and PPD were 3,010.00 (IQR 1,257.504,110.00), 610.00 (IQR 123.25-1,925.00) and 200.00 (IQR 42.80-1,580.00) $\mathrm{pg} \cdot \mathrm{mL}^{-1}$, respectively.

known ESAT-6 family proteins. These polypeptides were considered to be isoforms or homologous heteroforms of various ESAT-6 family proteins and were excluded. Thus, only polypeptides 36 (TB10.4), 38 (ESAT-6), 40 (CFP-8), 43(CFP-10) and 47 (CFP-15) were considered as five immunodominant polypeptides, predominantly recognised by healthy TB contacts on the basis of lymphoproliferative and IFN- $\gamma$ responses $(\mathrm{p}<0.5-<0.001)$ with respect to moderately advanced TB patients, suitable for inclusion in an MSV.

Conversely, when group-II polypeptides were analysed for utility in constituting experimental MSV, the order of recognition was $45>37>46>2>1>33>28$, considering both median SI $(>3.0)$ and IFN $-\gamma$ responses $\left(>50 \mathrm{pg} \cdot \mathrm{mL}^{-1}\right)$ induced by PBMCs of healthy TB contacts. However, polypeptide 45 reacted with IT-3/SA-12 mAb and showed cross reactivity with hsp 10, and was excluded. Similarly, polypeptide 37, which cross-reacted with mAbs used to identify known ESAT- 6 family proteins, was also not considered suitable for inclusion. Thus, polypeptides 46 (CFP-11), 2 (CFP-21), 1 (CFP-22.5), 33 (CFP-31) and 28 (MPT-64) from group II were considered suitable for development of an MSV. The characteristic feature of these polypeptides was that they all demonstrated subdominant lymphocyte proliferative responses (SI 3.06-5.36) compared to those from group I (SI 5.98-12.29). Barring polypeptide 2, the IFN- $\gamma$ responses of group-II polypeptides were also subdominant.

\section{Recognition of polypeptides in a healthy endemic population}

Group-I and group-II polypeptides were further analysed for recognition by T-lymphocytes of the general Indian population (fig 3). When the lymphoproliferative and IFN- $\gamma$ responses of immunodominant group-I antigens (polypeptides 36, 38, 40, 43 and 47) in healthy TB contacts (Fig. 1a and c) were compared to those observed in healthy individuals from the endemic population (fig. $3 a$ and $c$ ), the differences were found to be significant for all of these polypeptides $(\mathrm{p}<0.05-\mathrm{p}<0.001$ versus healthy endemic controls). These results demonstrate predominant recognition of polypeptides $36,38,40,43,47$ in healthy TB contacts compared to noncontacts. Subsequently, when the lymphoproliferative response of predominantly group-II antigens (i.e. 1, 2, 28, 33 and 46) in healthy TB contacts (fig. 1b) were compared with those observed in healthy individuals 

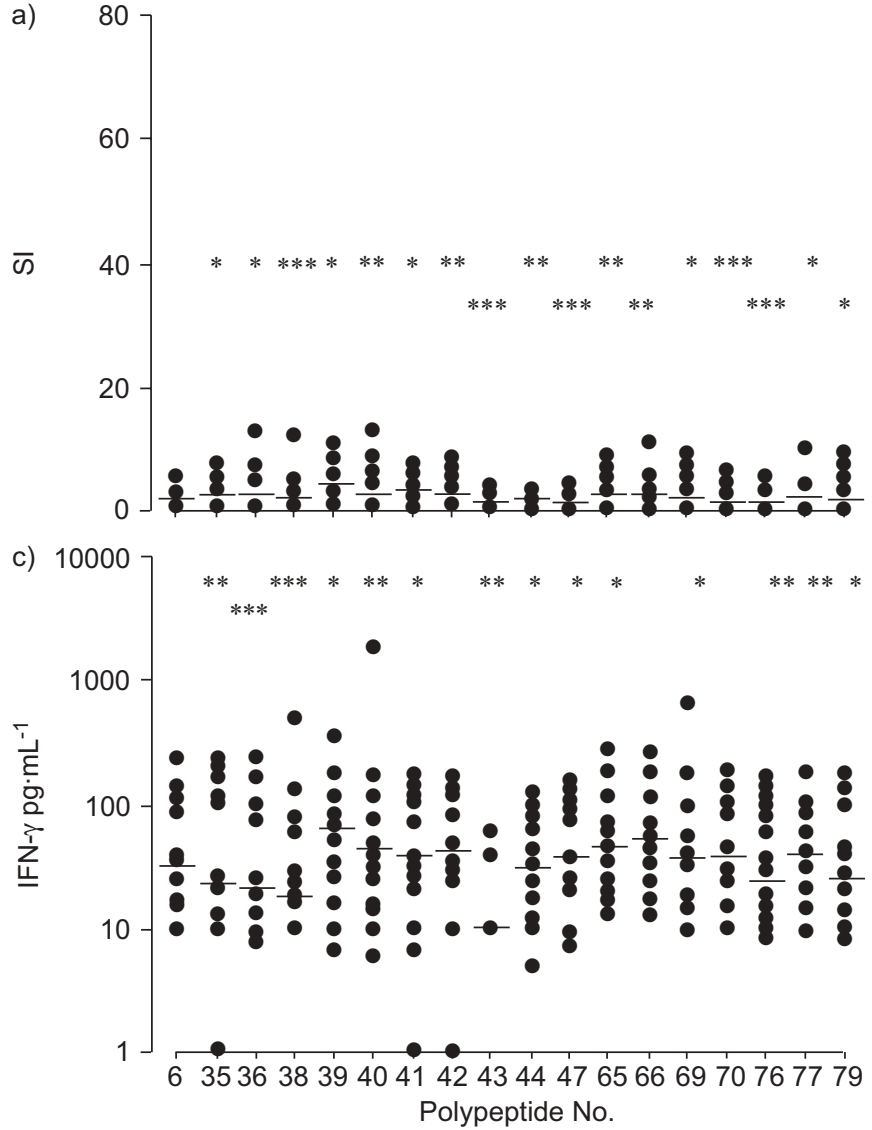

b)

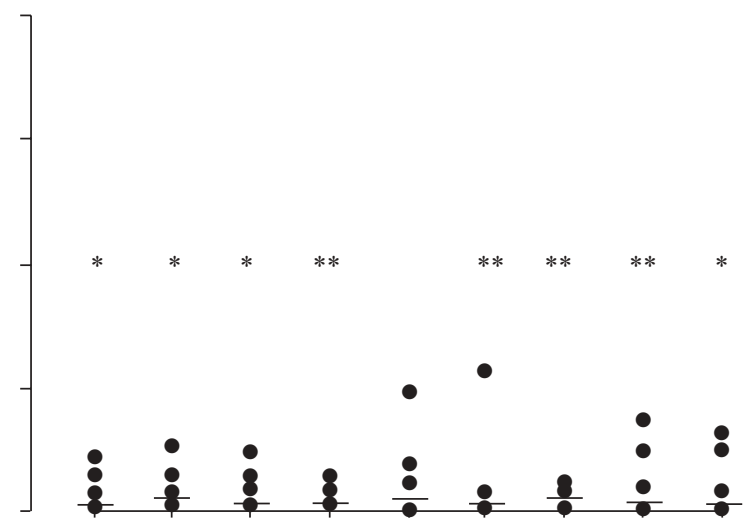

d)

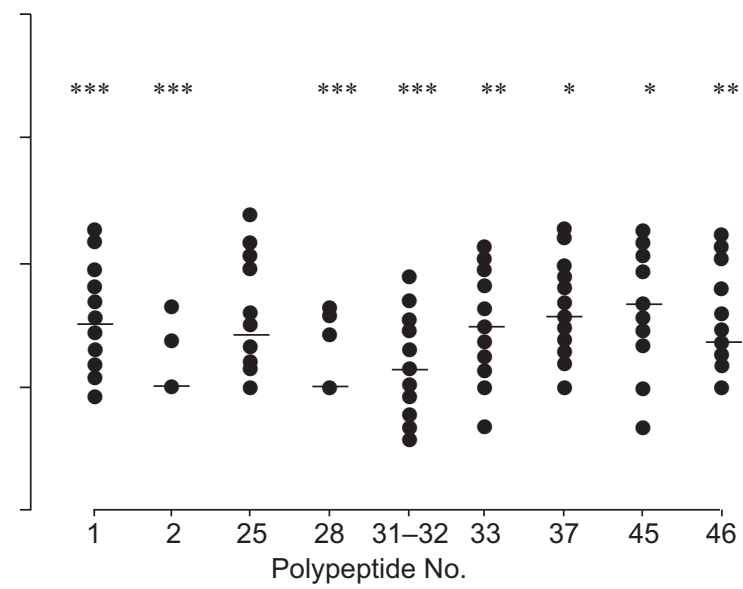

FIGURE 2. a, b) Lymphocyte proliferative and $c$, d) interferon (IFN)- $\gamma$ responses of moderately advanced tuberculosis (TB) patients ( $n=18)$ after in vitro stimulation with: a, c) group-I, and b, d) group-II low molecular mass purified polypeptides. Horizontal bars represent medians and each symbol represents one individual. The median counts per minute of cultures without antigen was 562. The median stimulation index (SI) in response to phytohaemagglutinin (PHA), total culture filtrate proteins of Mycobacterium tuberculosis H37Rv (RvCFP) and Siebert purified protein derivative of tuberculin (PPD) was 8.48 (interquartile range (IQR) 5.67-22.98), 3.22 (IQR 1.10-7.54) and 2.36 (IQR 1.42-3.64), respectively. The median IFN- $\gamma$ response of cultures without antigen was $8.0 \mathrm{pg} \cdot \mathrm{mL}^{-1}$. The IFN- $\gamma$ levels released into lymphocyte culture supernatants in response to in vitro stimulation with PHA, RvCFP and PPD were 490.00 (IQR 160.00-2,510.00), 10.00 (IQR 10.00-46.00) and 26.00 (IQR 10.00-52.00) pg. $\mathrm{mL}^{-1}$, respectively. *: p<0.05; $* *: p<0.01 ; * *: p<0.001$ versus healthy TB contacts.

from the endemic population (fig. 3b), the differences were nonsignificant for all of the above polypeptides. However, the IFN- $\gamma$ responses of all of the above group-II polypeptides were significantly high in healthy $\mathrm{TB}$ contacts compared to noncontacts (fig. $1 \mathrm{~d}$ and $3 \mathrm{~d}$ ).

\section{Cytotoxic T-cell response induced by mycobacterial antigens}

Antigens were also analysed for their ability to induce in vitro cytotoxic T-cell responses in healthy TB contacts (fig. 4). Amongst group I, only polypeptides 36 and 38 were recognised predominantly by CTLs (cytotoxicity $>30 \%$ ). Conversely, amongst immunodominant group-II polypeptides (i.e. 1, 2, 28, 33 and 46), predominant cytotoxicity was observed when macrophages were pulsed with polypeptides $2,28,33$ and 46 .

\section{Protective efficacy of experimental subunit vaccines}

TB10.4, ESAT-6, CFP-8, CFP-10 and CFP-15 were used to constitute experimental MSV-1, whereas CFP-21, CFP-22.5, MPT-64, CFP-31 and CFP-11 constituted MSV-2. Investigation of their protective efficacy, by determining the number of cfu in the lungs and spleens of mice, revealed significantly lower $\log _{10} \mathrm{cfu}$ in both of the experimental vaccine groups and the BCG-immunised group than in unvaccinated controls (table 3). MSV-2 imparted better protection than MSV-1, and the protection imparted was found to be significant at the level of spleen $(p<0.05)$. The protective efficacy of MSV-2 was, however, found to be comparable to that of BCG. These results demonstrate the utility of MSV-2 as a prospective MSV and needs further evaluation.

\section{DISCUSSION}

According to the existing notion, mycobacterial antigens inducing dominant cellular immune responses should be considered to be important for inclusion in future subunit antituberculous vaccines. However, this concept has been challenged by some recent reports indicating higher protective efficacy of T-cell-subdominant antigens compared to immunodominant antigens [18]. Further, in order to develop an ideal subunit vaccine, selected candidate antigens should be able to be recognised by a genetically diverse population, representing a broad spectrum of major histocompatibility complex molecules [12]. Therefore, selected mycobacterial proteins, inducing 
a)

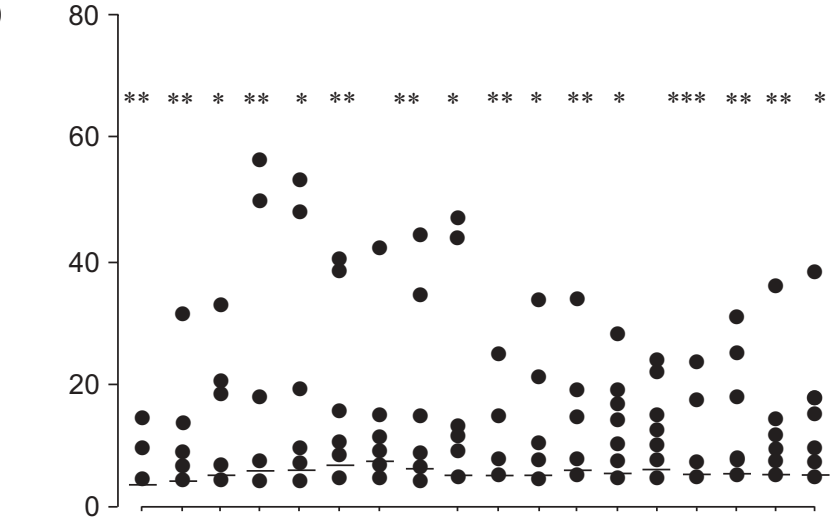

c) $100007 * * * * \quad * * * * * * * \quad * * \quad * * * \quad * * * * * * \quad * * * * * *$

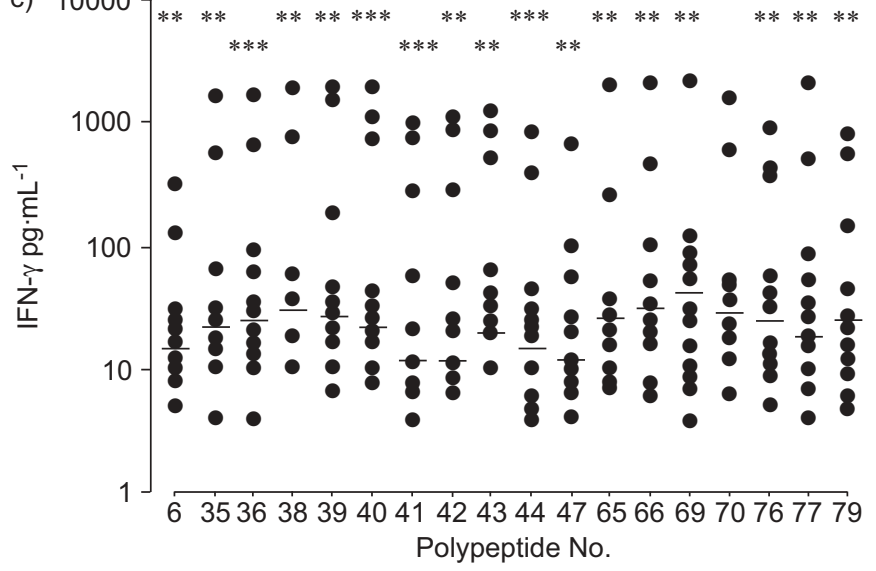

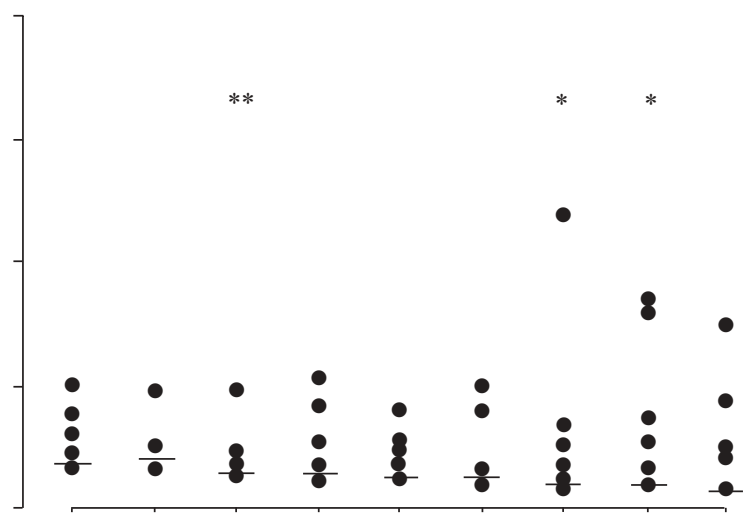

d)

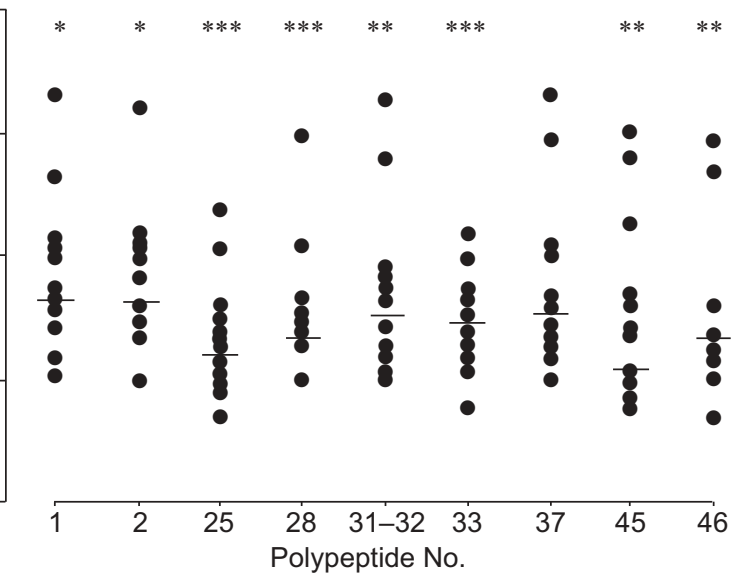

FIGURE 3. a, b) Lymphocyte proliferative and c, d) interferon (IFN)- $\gamma$ responses of healthy tuberculosis (TB) noncontacts ( $\mathrm{n}=17)$ after in vitro stimulation with: a, c) group-l, and b, d) group-II low molecular mass purified polypeptides. The median induration response in tuberculin skin tests carried out in the study subjects was $13 \mathrm{~mm}$. Horizontal bars represent medians and each symbol represents one individual. The median counts per minute of cultures without antigen was 726 . The median stimulation index (SI) in response to phytohaemagglutinin (PHA), total culture filtrate proteins of Mycobacterium tuberculosis H37Rv (RvCFP) and Siebert purified protein derivative of tuberculin (PPD) was 24.80 (interquartile range (IQR) 20.57-33.90), 4.22 (IQR 2.80-5.95) and 3.70 (IQR 2.03-5.60), respectively. The median IFN- $\gamma$ response of cultures without antigen was $4.00 \mathrm{pg} \cdot \mathrm{mL}^{-1}$. The IFN- $\gamma$ levels released into lymphocyte culture supernatants in response to in vitro stimulation with PHA, RvCFP and PPD were $3,840.00$ (IQR 3,800.00-4,085.00), 282.00 (IQR 144.00-660.00) and 79.50 (IQR 19.35-133.00) pg. $\mathrm{mL}^{-1}$, respectively. *: $p<0.05 ;{ }^{* *}$ : $p<0.01$; ***: $p<0.001$ versus healthy TB contacts.

T-cell-immunodominant (group-I) and -subdominant (group-II) responses, were evaluated for their recognition by different donor categories. The results of the present study clearly demonstrate that healthy TB contacts show a greatly enhanced response to the mycobacterial antigens tested, whereas $\mathrm{TB}$ patients exhibit depressed immune responses. These observations are in good agreement with the results of various previous studies [9-11]. Furthermore, observations of enhanced recognition of mycobacterial antigens in healthy contacts compared to noncontacts, as well as observations in healthy TB noncontacts (endemic controls) compared to TB patients, were also consistent with those reported earlier from endemic countries $[19,20]$. These recognition studies in different donor categories helped in the selection of suitable candidates from group I and group II for constitution of experimental MSVs. MSV-1 included five polypeptides inducing dominant T-cell-mediated immune responses, whereas MSV-2 included five polypeptides having subdominant T-cell immune responses along with antibody response.

When the two experimental MSVs, i.e. MSV-1 and MSV-2, selected on the basis of human recognition were compared for protective efficacy, MSV-2 imparted better protection at the level of both lungs and spleen. These findings indicate that no correlation exists between the magnitude of the in vitro Thelper cell (Th) type 1 response induced by an antigen during natural processing in healthy TB contacts and the extent of protection imparted by the same antigen after immunisation in a mouse model, comparable with the earlier observations of OLSEN et al. [18]. ESAT-6, CFP-10 and TB10.4, which constituted MSV-1, are predominantly expressed in virulent mycobacterial species and are considered virulence factors [21-23]. The possibility of these polypeptides acting as decoy antigens, diverting the immune response towards a biased Th1-type response, resulting in pathology, cannot be ruled out [24]. As many as $29 \%$ of healthy household contacts in whom in vitro ESAT-6-specific responses are induced were previously suspected to develop active TB [25]. Recently ESAT-6 and CFP-10 have been shown to form a 1:1 complex [26], leading to tissue destruction [27]. This might be one of the reasons why MSV-1 imparted less protection. Moreover, all of the polypeptides used in MSV-1 had a molecular mass of $<15 \mathrm{kDa}$ and were found to exhibit less inherent immunogenicity compared to the 

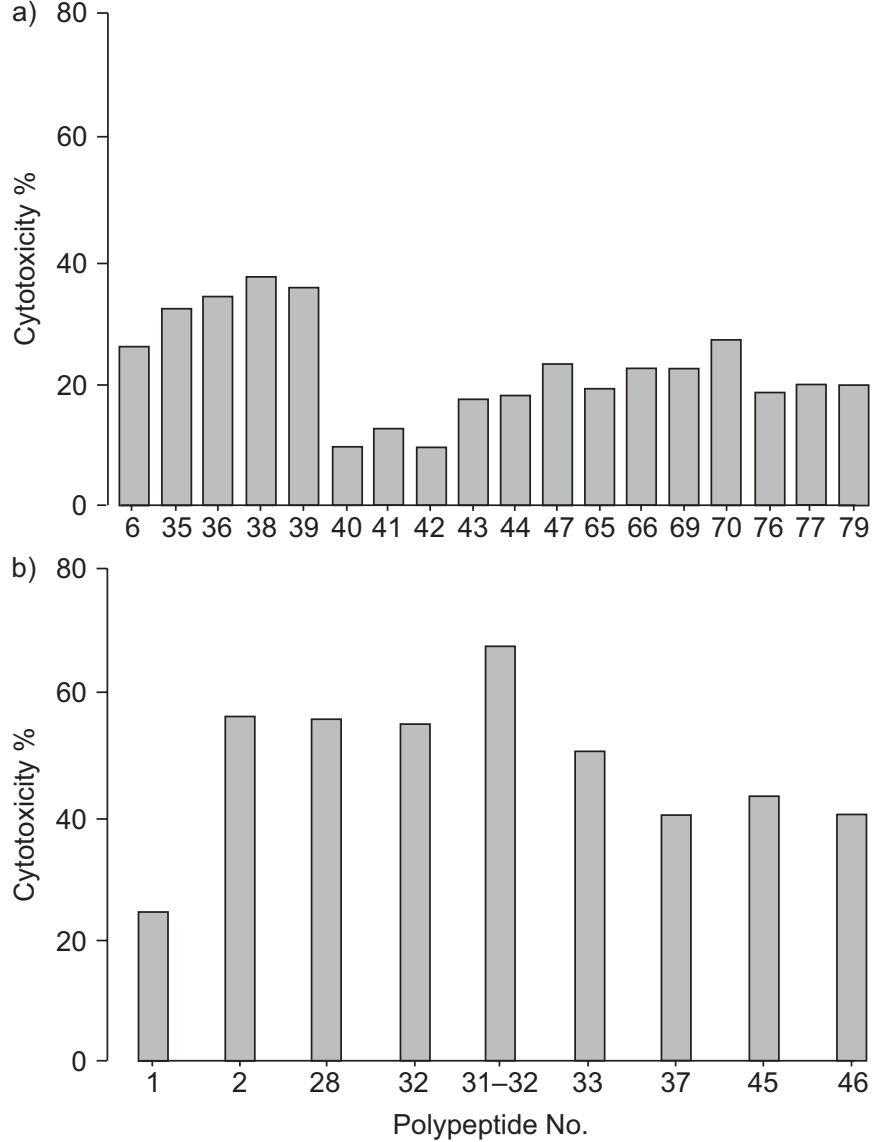

FIGURE 4. Evaluation of the ability of: a) group-l, and b) group-II low molecular mass purified polypeptides to induce autologous macrophage cytotoxicity in healthy tuberculosis contacts $(n=8)$. The median induration response in skin tests carried out in the study subjects was $23 \mathrm{~mm}$. The median percentage cytotoxicity of cultures without antigen was 7.08. Cytotoxicity was determined using the formula: percentage lysis $=100((C-B)-(E-B)) /(C-B)$, where $C$ is the mean optical density (OD) of macrophages without effector cells, $B$ is the mean OD of wells without cells and $E$ is the mean $O D$ of macrophages plus effector cells.

polypeptides used to constitute MSV-2, as determined in mice immunised with RvCFP-DDA-MPL (data not shown). Further, the increased protection imparted by MSV-2 might be due to an increased CTL response to the constitutive polypeptides (fig. 4). Another interesting feature of MSV-2 polypeptides is that all have previously been observed to produce a significant antibody response (data not shown). These results suggest a possible role of antibodies in protection against $M$. tuberculosis. It should be noted that Ag85B, previously defined as protective antigen, was among the group-II polypeptides in the present study and was found to augment both the cell-mediated and humoral immune response with Th1 and Th2 cytokine induction [28]. Moreover, the protective ESAT- $6^{51-71}$ peptide [18] has previously been shown to contain both T- and B-cell epitopes [29]. Recently, antibodies have also been shown to exhibit protective effect against TB [30, 31]. These results strengthen the emerging view that the most effective immune response is one that combines both humoral and cellular components for intracellular pathogens such as M. tuberculosis [30].

\begin{tabular}{|c|c|c|}
\hline \multirow[t]{2}{*}{ TABLE 3} & \multicolumn{2}{|c|}{$\begin{array}{l}\text { Mycobacterium tuberculosis H37Rv density } \\
4 \text { weeks after infection in the lungs and spleen of } \\
\text { vaccinated }{ }^{\#} \text { C57BL/6J mice }\end{array}$} \\
\hline & Lung $\log _{10} c f u$ & Spleen $\log _{10} \mathrm{cfu}$ \\
\hline Unvaccinated & $5.901 \pm 0.217$ & $5.744 \pm 0.193$ \\
\hline PBS-DDA/MPL & $5.764 \pm 0.223$ & $5.628 \pm 0.294$ \\
\hline BCG & $4.949 \pm 0.341 * *$ & $4.863 \pm 0.166^{\star \star}$ \\
\hline MSV-1 & $5.240 \pm 0.174^{\star \star}$ & $5.213 \pm 0.360^{*}$ \\
\hline MSV-2 & $4.978 \pm 0.384^{\star *}$ & $4.704 \pm 0.229^{\star \star \star}$ \\
\hline \multicolumn{3}{|c|}{ 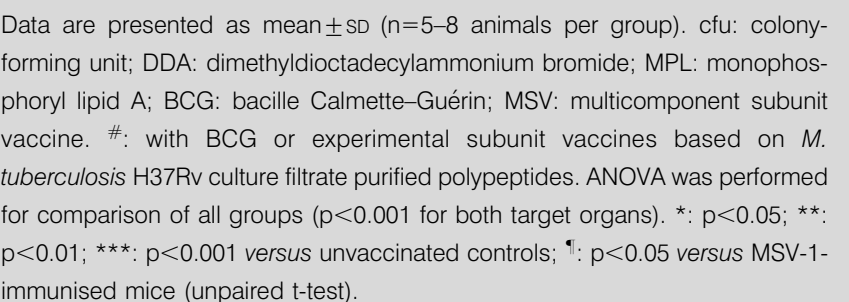 } \\
\hline
\end{tabular}

In the present study, contrary to the existing notion, significant protection is imparted by the polypeptides inducing subdominant interferon- $\gamma$ levels and substantial levels of antibody compared to that imparted by dominant interferon- $\gamma$-inducing polypeptides selected on the basis of human recognition during subclinical infection. The present observation, therefore, emphasises the need for careful selection of antigens for the constitution of experimental subunit vaccines against tuberculosis.

\section{ACKNOWLEDGEMENTS}

The authors would like to thank J.T. Belisle (Colorado State University, Fort Collins, CO, USA) and I. Rosenkrands (Statens Serum Institute, Copenhagen, Denmark) for providing low molecular mass, culture filtrate protein-specific monoclonal antibodies. The clinical assistance of V.B. Singla (Tuberculosis and Chest Diseases Hospital, Patiala, India) is gratefully acknowledged. The authors would also like to thank all of the volunteers who participated in the present study.

\section{REFERENCES}

1 Pal PG, Horwitz MA. Immunization with extracellular proteins of Mycobacterium tuberculosis induces cell mediated immune responses and substantial protective immunity in a guinea pig model of pulmonary tuberculosis. Infect Immun 1992; 60: 4781-4792.

2 Andersen P. Effective vaccination of mice against Mycobacterium tuberculosis infection with a soluble mixture of secreted mycobacterial proteins. Infect Immun 1994; 62: 2536-2544.

3 Horwitz MA, Lee BW, Dillon BJ, Harth G. Protective immunity against tuberculosis induced by vaccination with major extracellular proteins of Mycobacterium tuberculosis. Proc Natl Acad Sci USA 1995; 92: 1530-1534.

4 Brandt L, Elhay M, Rosenkrands I, Lindblad EB, Andersen P. ESAT-6 subunit vaccination against Mycobacterium tuberculosis. Infect Immun 2000; 68: 791-795. 
5 Coler RN, Campos-Neto A, Ovendale P, et al. Vaccination with $\mathrm{T}$ cell antigen Mtb 8.4 protects against challenge with Mycobacterium tuberculosis. J Immunol 2001; 166: 6227-6235.

6 Sinha RK, Verma I, Khuller GK. Immunobiological properties of $30 \mathrm{kDa}$ secretory protein of Mycobacterium tuberculosis $\mathrm{H}_{37} \mathrm{Ra}$. Vaccine 1997; 15: 689-699.

7 Sharma AK, Verma I, Tiwari R, Khuller GK. Adjuvant modulation of T-cell reactivity to $30 \mathrm{kDa}$ secretory protein of Mycobacterium tuberculosis $\mathrm{H}_{37} \mathrm{Rv}$ and its protective efficacy against experimental tuberculosis. I Med Microbiol 1999; 48: 757-763.

8 Olsen AW, Van Pinxten LAH, Okkels LM, Rasmussen PB, Andersen P. Protection in mice with a tuberculosis subunit vaccine based on a fusion protein of antigen $85 \mathrm{~B}$ and ESAT-6. Infect Immun 2001; 69: 2773-2778.

9 Boesen H, Jensen BN, Wilcke T, Andersen P. Human T cell responses to secreted antigen fraction of Mycobacterium tuberculosis. Infect Immun 1995; 3: 1491-1497.

10 Bhaskar S, Khanna SP, Mukherjee R. Isolation, purification and immunological characterization of novel low molecular weight protein antigen CFP6 from culture filtrate of $M$. tuberculosis. Vaccine 2000; 18: 2856-2866.

11 Demissie A, Ravn P, Olobo J, et al. T cell recognition of Mycobacterium tuberculosis culture filtrate fractions in tuberculosis patients and their household contacts. Infect Immun 1999; 67: 5967-5971.

12 A tuberculosis subunit vaccine based on a fusion protein of Ag85B and ESAT-6; broad human immune recognition and prolonged survival of TB infected mice and guinea pigs. In: Arend SM. Cellular immune responses to mycobacteria: towards specific immunodiagnosis and protection. PhD thesis. Dept of Infectious Diseases, Leiden University Medical Centre, Leiden, The Netherlands, 2002; pp. 77-97.

13 National Tuberculosis and Respiratory Disease Association. Diagnostic Standards and Classification of Tuberculosis. New York, NY, National Tuberculosis and Respiratory Disease Association, 1969.

14 Subrahmanyam D. Studies on the polyglycerolphosphatide of Mycobacterium tuberculosis. Can J Biochem Physiol 1964; 42: 1195-1201.

15 Nagai S, Wiker HG, Harboe M, Kinomoto M. Isolation and partial characterization of major protein antigens in the culture fluid of Mycobacterium tuberculosis. Infect Immun 1991; 59: 372-382.

16 Parish CR, Mullbacher A. Automated colorimetric assay for T cell cytotoxicity. J Immunol Methods 1983; 58: 225-237.

17 Camus JC, Pryor MJ, Medigue C, Cole ST. Re-annotation of the genome sequence of Mycobacterium tuberculosis H37Rv. Microbiology 2002; 148: 2967-2973.

18 Olsen AW, Hansen PR, Holm A, Andersen P. Efficient protection against Mycobacterium tuberculosis by vaccination with a single subdominant epitope from the ESAT-6 antigen. Eur J Immunol 2000; 30: 1724-1732.

19 Lalvani A, Nagvenkar P, Udwadia Z, et al. Enumeration of $\mathrm{T}$ cells specific for RD1-encoded antigens suggests a high prevalence of latent Mycobacterium tuberculosis infection in healthy urban Indians. J Infect Dis 2001; 183: 469-477.

20 Vekemans J, Lienhardt C, Sillah JS, et al. Tuberculosis contacts but not patients have higher gamma interferon resposnses to ESAT-6 than do community controls in the Gambia. Infect Immun 2001; 69: 6554-6557.

21 Skjøt RL, Oettinger T, Rosenkrands I, et al. Comparative evaluation of low molecular mass proteins from Mycobacterium tuberculosis identifies members of the ESAT-6 family as immunodominant T cell antigens. Infect Immun 2000; 68: 214-220.

22 Harboe M, Oettinger T, Wiker HG, Rosenkrands I, Andersen P. Evidence for occurrence of the ESAT-6 protein in Mycobacterium tuberculosis and virulent Mycobacterium bovis and for its absence in Mycobacterium bovis BCG. Infect Immun 1996; 64: 16-22.

23 Lewis KN, Liao R, Guinn KM, et al. Deletion of RD1 from Mycobacterium tuberculosis mimics bacille Calmette-Guérin attenuation. J Infect Dis 2003; 187: 117-123.

24 Vordermeier HM, Chambers MA, Cockle PJ, Whelan AO, Simmons J, Hewinson RG. Correlation of ESAT-6 specific gamma interferon production with pathology in cattle following Mycobacterium bovis BCG vaccination against experimental bovine tuberculosis. Infect Immun 2002; 70: 3026-3032.

25 Doherty TM, Demissie A, Olobo J, et al. Immune responses to the Mycobacterium tuberculosis-specific antigen ESAT-6 signal subclinical infection among contacts of tuberculosis patients. J Clin Microbiol 2002; 40: 704-706.

26 Renshaw PS, Panagiotidou P, Whelan A, et al. Conclusive evidence that the major T-cell antigens of the Mycobacterium tuberculosis complex ESAT-6 and CFP-10 form a tight, 1:1 complex and characterization of the structural properties of ESAT-6, CFP-10 and the ESAT-6CFP-10 complex: implications for pathogenesis and virulence. J Biol Chem 2002; 277: 21598-21603.

27 Hsu T, Hingley-Wilson SM, Chen B, et al. The primary mechanism of attenuation of bacillus Calmette-Guerin is a loss of secreted lytic function required for invasion of lung interstitial tissue. Proc Natl Acad Sci USA 2003; 100: 12420-12425.

28 Torres M, Herrera T, Villareal H, Rich EA, Sada E. Cytokine profiles for peripheral blood lymphocytes from patients with active pulmonary tuberculosis and healthy household contacts in response to the 30 kilodalton antigen of Mycobacterium tuberculosis. Infect Immun 1998; 66: 176-180.

29 Harboe M, Wiker HG, Ulvund G, et al. B-cell epitopes and quantification of the ESAT-6 protein of Mycobacterium tuberculosis. Infect Immun 1998; 66: 717-723.

30 Casadevall A. Antibody-mediated immunity against intracellular pathogens: two-dimensional thinking comes full circle. Infect Immun 2003; 71: 4225-4228.

31 Williams A, Reljic R, Naylor I, et al. Passive protection with immunoglobulin A antibodies against tuberculosis early infection of the lungs. Immunology 2004; 111: 328-333. 